Department of Econometrics and Business Statistics

http://business.monash.edu/econometrics-and-businessstatistics/research/publications

\title{
Forecast reconciliation: A geometric view with new insights on bias correction
}

Anastasios Panagiotelis, Puwasala Gamakumara, George Athanasopoulos and Rob J Hyndman

September 2019 


\title{
Forecast reconciliation: A geometric view with new insights on bias correction
}

\author{
Anastasios Panagiotelis \\ Department of Econometrics and Business Statistics, \\ Monash University, VIC 3145, Australia. \\ Email: Anastasios.Panagiotelis@monash.edu \\ Puwasala Gamakumara \\ Department of Econometrics and Business Statistics, \\ Monash University, VIC 3800, Australia. \\ Email: Puwasala.Gamakumara@monash.edu \\ George Athanasopoulos* \\ Department of Econometrics and Business Statistics, \\ Monash University, VIC 3145, Australia. \\ Email: George.Athanasopoulos@monash.edu \\ Rob J Hyndman \\ Department of Econometrics and Business Statistics, \\ Monash University, VIC 3800, Australia. \\ Email: Rob.Hyndman@monash.edu
}

September 15, 2019

\footnotetext{
*Corresponding Author. The authors gratefully acknowledge the support of Australian Research Council Grant DP140103220. We also thank Professor Mervyn Silvapulle for valuable comments.
} 


\begin{abstract}
A geometric interpretation is developed for so-called reconciliation methodologies used to forecast time series that adhere to known linear constraints. In particular, a general framework is established nesting many existing popular reconciliation methods within the class of projections. This interpretation facilitates the derivation of novel results that explain why and how reconciliation via projection is guaranteed to improve forecast accuracy with respect to a specific class of loss functions. The result is also demonstrated empirically. The geometric interpretation is further used to provide a new proof that forecast reconciliation results in unbiased forecasts provided the initial base forecasts are also unbiased. Approaches for dealing with biased base forecasts are proposed and explored in an extensive empirical study on Australian tourism flows. Overall, the method of bias-correcting before carrying out reconciliation is shown to outperform alternatives that only bias-correct or only reconcile forecasts.
\end{abstract}




\section{Introduction}

The past decade has seen rapid development in methodologies for forecasting time series that follow a hierarchical aggregation structure. Of particular prominence have been forecast reconciliation methods involving two steps: first separate forecasts are produced for all series, then these are adjusted ex post to ensure coherence with aggregation constraints. Forecast reconciliation has mostly been formulated using a regression model, see Hyndman et al. (2011) and Wickramasuriya et al. (2019) for examples. This setup can be counterintuitive since a vector comprised of forecasts from different time series models is also assumed to be the dependent variable in a regression model. In this paper, we eschew a regression interpretation in favour of a novel, geometric understanding of forecast reconciliation. This allows us to develop novel proofs and a clearer understanding of the interplay between forecast bias and reconciliation methods.

Multivariate time series following an aggregation structure arise in many sectors such as retail, energy, insurance, health and welfare and economics (see for example Karmy \& Maldonado 2019, Ben Taieb et al. 2017, Nystrup et al. 2019, Almeida et al. 2016, Jeon et al. 2019, Mahkya et al. 2017, Li \& Tang 2019, Shang \& Hyndman 2017, Athanasopoulos et al. 2019). Forecasts of these series should adhere to aggregation constraints to ensure aligned decision making. Earlier studies achieved this by only forecasting a single level of the hierarchy and then either aggregating in a bottom-up fashion (Dunn et al. 1976) or disaggregating in a top-down fashion (Gross \& Sohl 1990, Athanasopoulos et al. 2009). For reviews of these approaches, including a discussion of their advantages and disadvantages, see Schwarzkopf et al. (1988), Kahn (1998), Lapide (1998), Fliedner (2001).

In contrast to these methods, Hyndman et al. (2011) proposed forecasting all series in the hierarchy, referring to these as base forecasts. Since base forecasts were produced independently they were not guaranteed to adhere to aggregation constraints and could thus be improved via further adjustment. A framework was proposed whereby the aggregation constraints were expressed in a regression model for the base forecasts. The predicted values from this model were guaranteed to adhere to the linear constraints by construction and could thus be used as a new set of forecasts. This approach and later modifications have 
subsequently been shown to outperform bottom-up and top-down approaches in a variety of empirical settings (see for example Athanasopoulos et al. 2009, 2017, Wickramasuriya et al. 2019, among others). Some theoretical insight into the performance of forecast reconciliation methods has been provided by Van Erven \& Cugliari (2015) and Wickramasuriya et al. (2019). Both papers provide a proof that reconciliation is guaranteed to improve base forecasts. The latter paper also proposes a particular version of reconciliation known as the Minimum Trace (MinT) method. This is optimal in the sense of minimising the trace of the reconciled forecast error covariance matrix under the assumption that the base forecasts are unbiased.

Our main contribution is to propose a geometric interpretation of the entire hierarchical forecasting problem. In this setting, we show that reconciled forecasts have a number of attractive properties when they are obtained via projections. We believe that this is clearer and more intuitive than explanations based on regression modelling. In addition to casting existing results in a new light, the geometric interpretation also allows us to derive three new important results.

First, our approach makes it clear that the defining characteristic of so-called hierarchical time series is not aggregation but linear constraints. As a result forecast reconciliation can be applied in contexts where there are no clear candidates of bottom level series, an insight that is not apparent when the problem is viewed through the lens of regression modelling. Second, we provide a new proof that reconciled forecasts dominate unreconciled forecasts which makes explicit the link between a reconciliation method and a loss function. We believe that this link is lacking in previous work that attempts to establish similar results, in particular Van Erven \& Cugliari (2015) and Wickramasuriya et al. (2019). Futhermore, unlike Van Erven \& Cugliari (2015) and Wickramasuriya et al. (2019), our proof does not require an assumption about convexity that may not hold in general. Third, we prove that reconciliation using certain projection matrices guarantees unbiased reconciled forecasts provided the base forecasts are also unbiased. A natural question that arises is what to do in the case of biased reconciled forecasts. Rather than addressing this issue by considering matrices that are not projections, we propose to bias-correct before 
reconciliation. This is evaluated in an extensive empirical study where we find that even when bias correction fails, the extent of the problem is mitigated by reconciling forecasts.

The remainder of this paper is structured as follows. Section 2 deals with the concept of coherence and defines hierarchical time series in a way that does not depend on any notion of bottom-level series. Section 3 defines forecast reconciliation in terms of projections and includes a proof that reconciled forecasts dominate base forecasts with respect to a specific loss function. In Section 4 we prove the unbiasedness preserving property of reconciliation via certain projection matrices and propose methods for bias correction. In Section 5 we conduct an extensive empirical application to domestic tourism flow in Australia with two objectives; first to demonstrate the theorems discussed in Section 3, second to evaluate the methods for bias correction discussed in Section 4. Section 6 concludes with some discussion and thoughts on the future research directions forf forecast reconciliation.

\section{Coherent forecasts}

\subsection{Notation and preliminaries}

We briefly define the concept of a hierarchical time series in a fashion similar to Athanasopoulos et al. (2019), Hyndman \& Athanasopoulos (2018) and others, before elaborating on some of the limitations of this understanding. A hierarchical time series is a collection of $n$ variables indexed by time, where some variables are aggregates of other variables. We let $\boldsymbol{y}_{t} \in \mathbb{R}^{n}$ be a vector comprising observations of all variables in the hierarchy at time $t$. The bottom-level series are defined as those $m$ variables that cannot be formed as aggregates of other variables; we let $\boldsymbol{b}_{t} \in \mathbb{R}^{m}$ be a vector comprised of observations of all bottom-level series at time $t$. The hierarchical structure of the data implies that the following holds for all $t$ :

$$
\boldsymbol{y}_{t}=\boldsymbol{S} \boldsymbol{b}_{t}
$$

where $\boldsymbol{S}$ is an $n \times m$ constant matrix that encodes the aggregation constraints. 


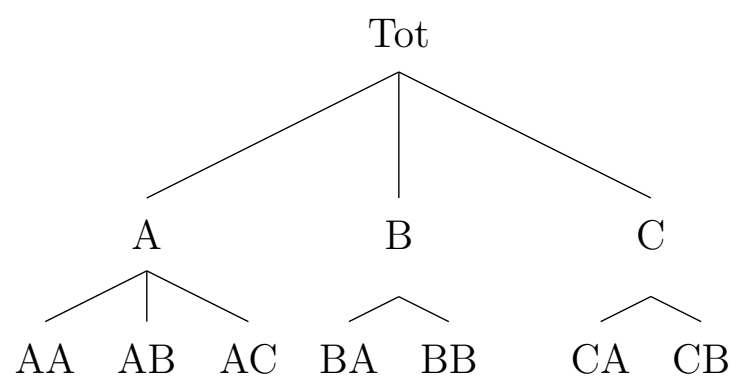

Figure 1: An example of a two level hierarchical structure.

To clarify these concepts, consider the example of the hierarchy in Figure 1. For this hierarchy, $n=11, \boldsymbol{y}_{t}=\left[y_{T o t, t}, y_{A, t}, y_{B, t}, y_{C, t}, y_{A A, t}, y_{A B, t}, y_{A C, t}, y_{B A, t}, y_{B B, t}, y_{C A, t}, y_{C B, t}\right]^{\prime}$, $m=7, \boldsymbol{b}_{t}=\left[y_{A A, t}, y_{A B, t}, y_{A C, t}, y_{B A, t}, y_{B B, t}, y_{C A, t}, y_{C B, t}\right]^{\prime}$ and

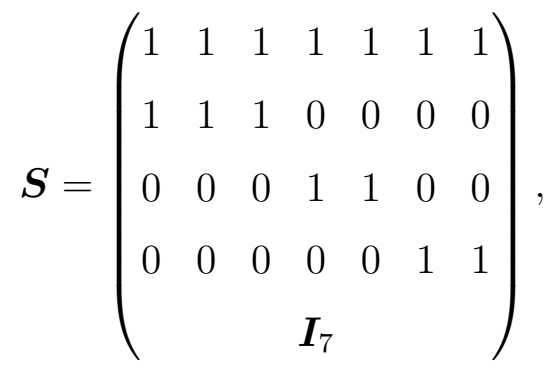

where $\boldsymbol{I}_{7}$ is the $7 \times 7$ identity matrix.

While such a definition is completely serviceable, it obscures the full generality of the literature on so-called hierarchical time series. In fact, concepts such as coherence and reconciliation, defined in full below, require the data to have only two important characteristics: the first is that they are multivariate, the second is that they adhere to linear constraints.

\subsection{Coherence}

The property that data adhere to some linear constraints is referred to as coherence. We now provide definitions aimed at providing geometric intuition of hierarchical time series.

Definition 2.1 (Coherent subspace). The $m$-dimensional linear subspace $\mathfrak{s} \subset \mathbb{R}^{n}$ for which some linear constraints hold for all $\boldsymbol{y} \in \mathfrak{s}$ is defined as the coherent subspace. 
To further illustrate, Figure 2 depicts the simplest three variable hierarchy where $y_{\text {Tot, } t}=$ $y_{A, t}+y_{B, t}$. The coherent subspace is depicted as a grey 2-dimensional plane within 3dimensional space; i.e. $m=2$ and $n=3$. It is worth noting that the coherent subspace is spanned by the columns of $\boldsymbol{S}$; i.e. $\mathfrak{s}=\operatorname{span}(\boldsymbol{S})$. In Figure 2 , these columns are $\vec{s}_{1}=(1,1,0)^{\prime}$ and $\vec{s}_{2}=(1,0,1)^{\prime}$. However, it is equally important to recognise that the hierarchy could also have been defined in terms of $y_{T o t, t}$ and $y_{A, t}$ rather than the bottom-level series, $y_{A, t}$ and $y_{B, t}$. In this case the corresponding ' $\boldsymbol{S}$ matrix' would have columns $(1,0,1)^{\prime}$ and $(0,1,-1)^{\prime}$. However, while there are multiple ways to define an $\boldsymbol{S}$ matrix, in all cases the columns will span the same coherent subspace, which is unique.

Definition 2.2 (Hierarchical Time Series). A hierarchical time series is an $n$-dimensional multivariate time series such that all observed values $\boldsymbol{y}_{1}, \ldots, \boldsymbol{y}_{T}$ and all future values $\boldsymbol{y}_{T+1}, \boldsymbol{y}_{T+2}, \ldots$ lie in the coherent subspace, i.e., $\boldsymbol{y}_{t} \in \mathfrak{s} \quad \forall t$.

Despite the common use of the term hierarchical time series, it should be clear from the definition that the data need not necessarily follow a hierarchy. Also notable by its absence in the above definition is any reference to aggregation. In some ways, terms such as hierarchical and aggregation can be misleading since the literature has covered instances that cannot be depicted in a similar fashion to Figure 1 and/or do not involve aggregation. Examples include, temporal hierarchies which involve grouped structures (see Athanasopoulos et al. 2017), overlapping temporal hierarchies (see Jeon et al. 2019), applications for which the difference rather than the aggregate is of interest (see Li \& Tang 2019), or structures that involve both cross-sectional and temporal dimensions referred to as cross-temporal structures (see Kourentzes \& Athanasopoulos 2019). Finally, although Definition 2.2 makes reference to time series, this definition can be easily generalised to any vector-valued data for which some linear constraints are known to hold for all realisations.

Definition 2.3 (Coherent Point Forecasts). Let $\breve{\boldsymbol{y}}_{t+h \mid t} \in \mathbb{R}^{n}$ be a vector of point forecasts of all series in the hierarchy where the subscript $t+h \mid h$ implies that the forecast is made as time $t$ for a period $h$ steps into the future. Then $\breve{\boldsymbol{y}}_{t+h \mid t}$ is coherent if $\breve{\boldsymbol{y}}_{t+h \mid t} \in \mathfrak{s}$.

Without any loss of generality, the above definition could also be applied to prediction for multivariate data in general, rather than just forecasting of time series. 


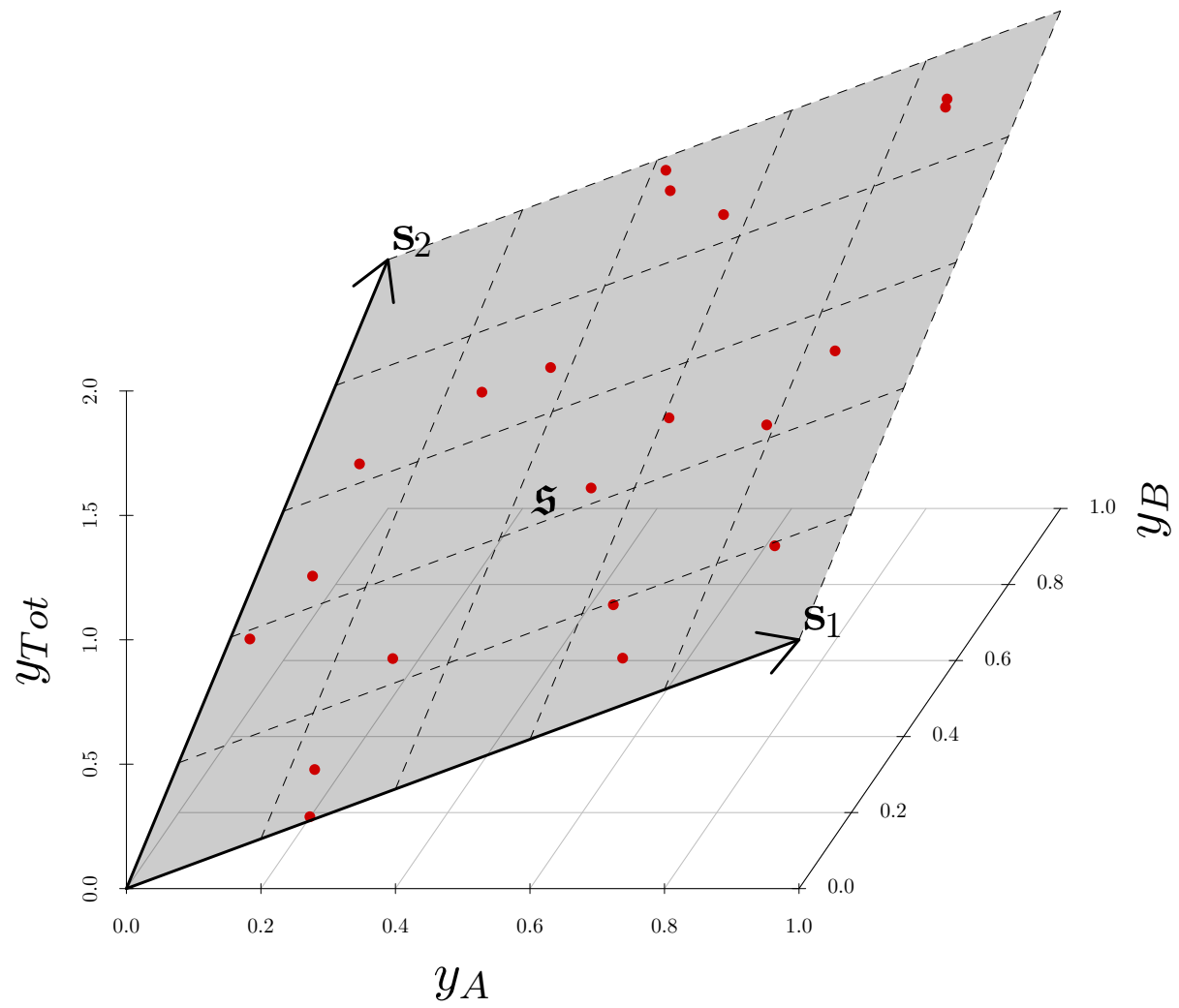

Figure 2: Depiction of a three dimensional hierarchy with $y_{\text {Tot }}=y_{\mathrm{A}}+y_{\mathrm{B}}$. The gray coloured two dimensional plane depicts the coherent subspace $\mathfrak{s}$ where $\vec{s}_{1}=(1,1,0)^{\prime}$ and $\vec{s}_{2}=(1,0,1)^{\prime}$ are basis vectors that span $\mathfrak{s}$. The red points in $\mathfrak{s}$ represent realisations or coherent forecasts

Much of the early literature that dealt with the problem of forecasting hierarchical time series (see Gross \& Sohl 1990, and references therein) produced forecasts at a single level of the hierarchy in the first stage. Subsequently forecasts for all series were recovered through aggregation, disaggregation according to historical or forecast proportions, or some combination of both. Consequently, incoherent forecasts were not a problem in these earlier papers.

Forecasting a single level of the hierarchy did not, however echo common practice within many industries. In many organisations different departments or 'silos' each produced their 
own forecasts, often with their own information sets and judgemental adjustments. ${ }^{1}$ This approach does have several advantages over only forecasting a single level. First, there is no loss of information since all levels and series are modelled. Second, modelling higher level series often identifies features such as trend and seasonality that cannot be detected in noisy disaggregate data. However, when forecasts are produced independently at all levels, forecasts are likely to be incoherent. ${ }^{2}$ This problem of incoherent forecasts cannot in general be solved by multivariate modelling either. Instead, the solution is to make an ex post adjustment that ensures coherence, a process known as forecast reconciliation

\section{Forecast reconciliation}

The concept of forecast reconciliation is predicated on there being an $n$-vector of forecasts that are incoherent. We call these base forecasts and denote them as $\hat{\boldsymbol{y}}_{t+h \mid t}$. In the sequel, this subscript will be dropped at times for ease of exposition. In the most general terms, reconciliation can be defined as follows.

Definition 3.1 (Reconciled forecasts). Let $\psi$ be a mapping, $\psi: \mathbb{R}^{n} \rightarrow \mathfrak{s}$. The point forecast $\tilde{\boldsymbol{y}}_{t+h \mid t}=\psi\left(\hat{\boldsymbol{y}}_{t+h \mid t}\right)$ "reconciles" a base forecast $\hat{\boldsymbol{y}}_{t+h \mid t}$ with respect to the mapping $\psi($.

All reconciliation methods that we are aware of consider a linear mapping for $\psi$, which involves pre-multiplying base forecasts by an $n \times n$ matrix that has $\mathfrak{s}$ as its image. One way to achieve this is with a matrix $\boldsymbol{S} \boldsymbol{G}$, where $\boldsymbol{G}$ is an $m \times n$ matrix (some authors use $\boldsymbol{P}$ in place of $\boldsymbol{G})$. This facilitates an interpretation of reconciliation as a two-step process. In the first step, base forecasts $\hat{\boldsymbol{y}}_{t+h \mid t}$ are combined to form a new set of bottom-level forecasts. In the second step, these are mapped to a full vector of coherent forecasts via pre-multiplication by $\boldsymbol{S}$.

Although pre-multiplying base forecasts by $\boldsymbol{S} \boldsymbol{G}$ will result in coherent forecasts, a number of desirable properties arise when $\boldsymbol{S G}$ has the specific structure of a projection ma-

\footnotetext{
${ }^{1}$ Chase (2013) discusses silos and the importance of information and data sharing across an organisation.

${ }^{2}$ There are some special cases of using simple approaches such as naïve, which extrapolate the coherent nature of the data to the forecasts.
} 
trix onto $\mathfrak{s}$. In general a projection matrix is defined via its idempotence property, i.e. $(\boldsymbol{S G})^{2}=\boldsymbol{S} \boldsymbol{G}$. However we also rely on another property of projection matrices, namely that any vector lying in the image of a projection is mapped to itself by that projection (see Lemma 2.4 in Rao 1974, for a proof). In our context this implies that for any $\boldsymbol{v} \in \mathfrak{s}$, $S G \boldsymbol{v}=\boldsymbol{v}$.

We begin by considering the special case of an orthogonal projection whereby $\boldsymbol{G}=$ $\left(\boldsymbol{S}^{\prime} \boldsymbol{S}\right)^{-1} \boldsymbol{S}^{\prime}$. This is equivalent to so called OLS reconciliation as introduced by Hyndman et al. (2011). We refrain from any discussion of regression models focusing instead on geometric interpretations. However the connection between OLS and orthogonal projection should be clear, in the context of regression modelling predicted values from OLS are obtained via an orthogonal projection of the response onto the span of the regressors.

\subsection{Orthogonal projection}

In this section we discuss two sensible properties that can be achieved by reconciliation via orthogonal projection.

- The first is that reconciliation should adjust the base forecasts as little as possible; i.e. the base and reconciled forecasts should be 'close'.

- The second is that reconciliation in some sense should improve forecast accuracy, or more loosely, that the reconciled forecast should be 'closer' to the realised value targeted by the forecast.

To address the first of these properties we make the concept of closeness more concrete, by considering the Euclidean distance between the base forecast $\hat{\boldsymbol{y}}$ and the reconciled forecast $\tilde{\boldsymbol{y}}$. A property of an orthogonal projection is that the distance between $\hat{\boldsymbol{y}}$ and $\tilde{\boldsymbol{y}}$ is minimal over any possible $\tilde{\boldsymbol{y}} \in \mathfrak{s}$. In this sense reconciliation via orthogonal projection leads to the smallest possible adjustments of the base forecasts.

The property that reconciliation should improve forecasts was touched upon in Section 2.3 of Wickramasuriya et al. (2019). The discussion in that paper focuses on the case of MinT. Here we provide a new explicit proof of that result. We do so first in the case 
of an orthogonal projection where the geometric intuition of the proof is clear and then generalise the result to reconciliation using any projection matrix in Section 3.2.

Consider the Euclidean distance between the target and a forecast. This is equivalent to the square root of the sum of squared forecast errors over the entire hierarchy. Let $\boldsymbol{y}_{t+h}$ be the realisation of the data generating process at time $t+h$. The following theorem shows that reconciliation never increases, the sum of squared errors of point forecasts.

Theorem 3.1 (Distance reducing property). If $\tilde{\boldsymbol{y}}_{t+h \mid t}=\boldsymbol{S} \boldsymbol{G} \hat{\boldsymbol{y}}_{t+h \mid t}$, where $\boldsymbol{G}$ is such that $\boldsymbol{S G}$ is an orthogonal projection (in the Euclidean sense) onto $\mathfrak{s}$ and let $\|\boldsymbol{v}\|$ be the $L_{2}$ norm (in the Euclidean sense) of vector $\boldsymbol{v}$ then:

$$
\left\|\left(\boldsymbol{y}_{t+h}-\tilde{\boldsymbol{y}}_{t+h \mid t}\right)\right\| \leq\left\|\left(\boldsymbol{y}_{t+h}-\hat{\boldsymbol{y}}_{t+h \mid t}\right)\right\|
$$

Proof. Since $\boldsymbol{y}_{t+h \mid t}, \tilde{\boldsymbol{y}}_{t+h \mid t} \in \mathfrak{s}$ and since the projection is orthogonal, by Pythagoras' theorem

$$
\left\|\left(\boldsymbol{y}_{t+h}-\hat{\boldsymbol{y}}_{t+h \mid t}\right)\right\|^{2}=\left\|\left(\boldsymbol{y}_{t+h}-\tilde{\boldsymbol{y}}_{t+h \mid t}\right)\right\|^{2}+\left\|\left(\tilde{\boldsymbol{y}}_{t+h \mid t}-\hat{\boldsymbol{y}}_{t+h \mid t}\right)\right\|^{2}
$$

Since $\left\|\left(\tilde{\boldsymbol{y}}_{t+h \mid t}-\hat{\boldsymbol{y}}_{t+h \mid t}\right)\right\|^{2} \geq 0$ this implies,

$$
\left\|\left(\boldsymbol{y}_{t+h}-\hat{\boldsymbol{y}}_{t+h \mid t}\right)\right\|^{2} \geq\left\|\left(\boldsymbol{y}_{t+h}-\tilde{\boldsymbol{y}}_{t+h \mid t}\right)\right\|^{2}
$$

with equality only holding when $\tilde{\boldsymbol{y}}_{t+h \mid t}=\hat{\boldsymbol{y}}_{t+h \mid t}$. Taking the square root of both sides proves the desired result.

The simple geometric intuition behind the proof is demonstrated in Figure 3. In this schematic, the coherent subspace is depicted as a black arrow, and the base forecast $\hat{\boldsymbol{y}}$ is shown as a blue dot. Since $\hat{\boldsymbol{y}}$ is incoherent, $\hat{\boldsymbol{y}}_{t+h \mid t} \notin \mathfrak{s}$ and in this case the inequality is strict. Reconciliation is an orthogonal projection from $\hat{\boldsymbol{y}}$ to the coherent subspace yielding the reconciled forecast $\tilde{\boldsymbol{y}}$ shown in red. Finally, the target of the forecast $\boldsymbol{y}$ is displayed as a black point, and although its exact location is unknown to the forecaster, it is known that it will lie somewhere along the coherent subspace.

Figure 3 clearly shows that $\hat{\boldsymbol{y}}, \tilde{\boldsymbol{y}}$ and $\boldsymbol{y}$ form a right angled triangle with $\tilde{\boldsymbol{y}}$ at the rightangled vertex. In this triangle the line between $\boldsymbol{y}$ and $\hat{\boldsymbol{y}}$ is the hypotenuse and therefore 


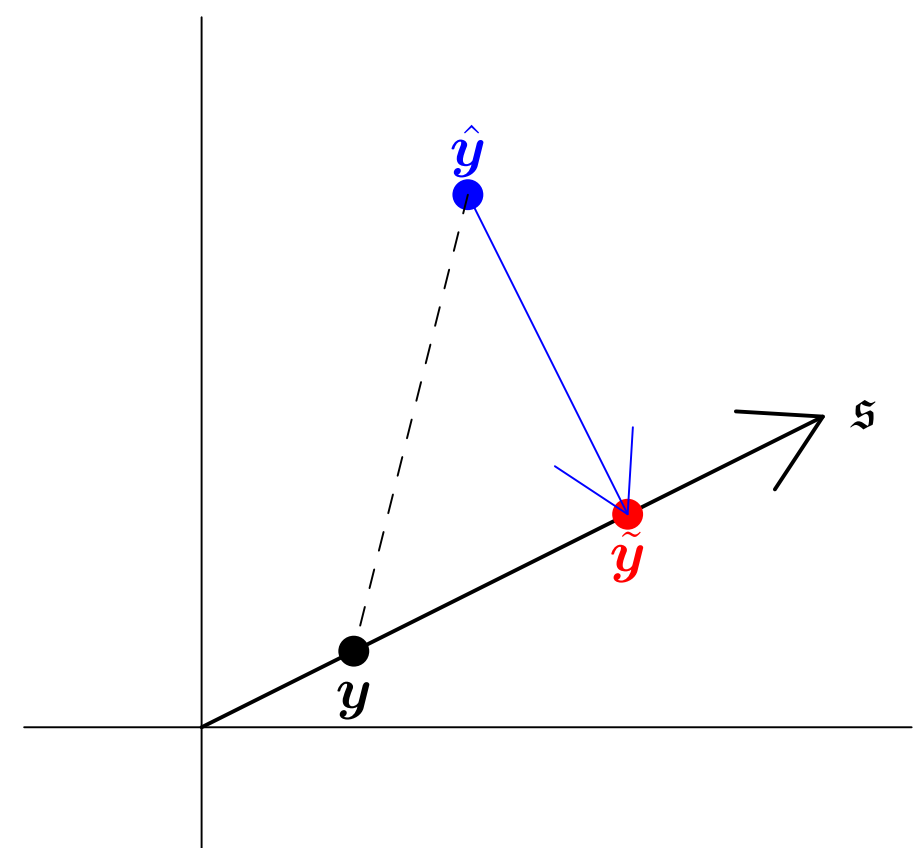

Figure 3: Orthogonal projection of $\hat{\boldsymbol{y}}$ onto $\mathfrak{s}$ yielding the reconciled forecast $\tilde{\boldsymbol{y}}$.

must be longer than the distance between $\boldsymbol{y}$ and $\tilde{\boldsymbol{y}}$. Therefore reconciliation is guaranteed to reduce the squared error of the forecast.

Theorem 3.1 is in some ways more powerful than perhaps previously understood. Crucially, the result is not a result that requires taking expectations. This distance reducing property will hold for any realisation and any forecast and not just on average. Nothing needs to be assumed about the statistical properties of the data generating process or the process by which forecasts are made.

However, in other ways, Theorem 3.1 is weaker than perhaps often understood. First, when improvements in forecast accuracy are discussed in the context of the theorem, this refers to a very specific measure of forecast accuracy. In particular, this measure is the square root of the sum of squared forecast errors of all variables in the hierarchy. Consequently, while forecast improvement is guaranteed for the hierarchy overall, reconciliation can lead to less accurate forecasts for individual series. Second, although orthogonal projections are guaranteed to improve on base forecasts, they are not necessarily the projection 
that leads to the greatest improvement in forecast accuracy. Therefore, referring to reconciliation via orthogonal projections as 'optimal' is somewhat misleading since it does not have the optimality properties of some oblique projections, in particular MinT. It is to oblique projections that we now turn our attention.

\subsection{Oblique Projections}

One justification for using an orthogonal projection is that it leads to improved forecast accuracy in terms of the square root of the sum of squared errors of all variables in the hierarchy. A clear shortcoming of this measure of forecast accuracy is that forecast errors in all series should not necessarily be treated equally. For example, in hierarchies, top-level series tend to have a much larger scale than bottom-level series. Even when two series are on a similar scale, series that are more predictable or less variable will tend to be downweighted by simply aggregating squared errors. An even more sophisticated understanding may take the correlation between series into account. All of these considerations lead towards reconciliation of the form $\tilde{\boldsymbol{y}}=\boldsymbol{S}\left(\boldsymbol{S}^{\prime} \boldsymbol{W}^{-1} \boldsymbol{S}\right)^{-1} \boldsymbol{S}^{\prime} \boldsymbol{W}^{-1} \hat{\boldsymbol{y}}$, where $\boldsymbol{W}$ is a symmetric matrix. Generally, it is assumed that $\boldsymbol{W}$ is invertible, otherwise a pseudo inverse can be used.

It should be noted that $\boldsymbol{S}\left(\boldsymbol{S}^{\prime} \boldsymbol{W}^{-1} \boldsymbol{S}\right)^{-1} \boldsymbol{S}^{\prime} \boldsymbol{W}^{-1}$ is an oblique, rather than an orthogonal projection matrix in the usual Euclidean geometry. However this matrix can be considered to be an orthogonal projection for a different geometry defined by the norm $\|\boldsymbol{v}\|_{\boldsymbol{W}^{-1}}=\boldsymbol{v}^{\prime} \boldsymbol{W}^{-1} \boldsymbol{v}$, referred to as the generalised Euclidean geometry with respect to $\boldsymbol{W}^{-1}$. One way to understand this geometry is that it is the same as Euclidean geometry when all vectors are first transformed by pre-multiplying by $\boldsymbol{W}^{-1 / 2}$. This leads to a transformed $\boldsymbol{S}$ matrix $\boldsymbol{S}^{*}=\boldsymbol{W}^{-1 / 2} \boldsymbol{S}$ and transformed $\hat{\boldsymbol{y}}$ and $\tilde{\boldsymbol{y}}$ vectors $\hat{\boldsymbol{y}}^{*}=\boldsymbol{W}^{-1 / 2} \hat{\boldsymbol{y}}$ and $\tilde{\boldsymbol{y}}^{*}=\boldsymbol{W}^{-1 / 2} \tilde{\boldsymbol{y}}$. The transformed reconciled forecast results from an orthogonal projection in the transformed space since

$$
\begin{aligned}
\tilde{\boldsymbol{y}}^{*} & =\boldsymbol{W}^{-1 / 2} \tilde{\boldsymbol{y}} \\
& =\boldsymbol{W}^{-1 / 2} \boldsymbol{S}\left(\boldsymbol{S}^{\prime} \boldsymbol{W}^{-1} \boldsymbol{S}\right)^{-1} \boldsymbol{S}^{\prime} \boldsymbol{W}^{-1} \hat{\boldsymbol{y}} \\
& =\boldsymbol{S}^{*}\left(\boldsymbol{S}^{*^{\prime}} \boldsymbol{S}^{*}\right)^{-1} \boldsymbol{S}^{*^{\prime}} \hat{\boldsymbol{y}}^{*}
\end{aligned}
$$


Thinking of the problem in terms of a geometry defined by the norm $\boldsymbol{v}^{\prime} \boldsymbol{W}^{-1} \boldsymbol{v}$ is also quite instructive when it comes to thinking about the connection between distances and loss functions. In the generalised Euclidean geometry, the distance between the reconciled forecast and the realisation is given by $(\boldsymbol{y}-\hat{\boldsymbol{y}})^{\prime} \boldsymbol{W}^{-1}(\boldsymbol{y}-\hat{\boldsymbol{y}})$. For diagonal $\boldsymbol{W}^{-1}$, this is equivalent to a weighted sum of squared error loss function and when $\boldsymbol{W}$ is a covariance matrix, this is equivalent to a Mahalanobis distance. Therefore Theorem 3.1 can easily be generalised as follows.

Theorem 3.2 (General distance reducing property). If $\tilde{\boldsymbol{y}}_{t+h \mid t}=\boldsymbol{S} \boldsymbol{G} \hat{\boldsymbol{y}}_{t+h \mid t}$, where $\boldsymbol{G}$ is such that $\boldsymbol{S G}$ is an orthogonal projection (in the generalised Euclidean sense) onto $\mathfrak{s}$ then:

$$
\left\|\left(\boldsymbol{y}_{t+h}-\tilde{\boldsymbol{y}}_{t+h \mid t}\right)\right\|_{\boldsymbol{W}^{-1}} \leq \mid\left(\boldsymbol{y}_{t+h}-\hat{\boldsymbol{y}}_{t+h \mid t}\right) \|_{\boldsymbol{W}^{-1}}
$$

Proof. The proof is identical to the proof for Theorem 3.1 but relies on the Generalised Pythagorean Theorem (applicable to Generalised Euclidean space) rather than the Pythagorean Theorem.

The implication of Theorem 3.2 is that if the objective function is some weighted sum of squared errors, or a Mahalanobis distance, then the projection matrix $\boldsymbol{S}\left(\boldsymbol{S}^{\prime} \boldsymbol{W}^{-1} \boldsymbol{S}\right)^{-1} \boldsymbol{S}^{\prime} \boldsymbol{W}^{-1}$ is guaranteed to improve forecast accuracy over base forecasts, for an appropriately selected $\boldsymbol{W}$.

Note here that we rely here on the Generalised Pythagorean Theorem (which involves an equality). In contrast, Wickramasuriya et al. (2019) follow Van Erven \& Cugliari (2015) in stating their result in terms of the Generalised Pythagorean Inequality. The proof of Wickramasuriya et al. (2019) requires an assumption about convexity so that the angle between the base forecast and coherent subspace must be greater than 90 degrees. The proof we have provided here requires no such assumption, since this may not hold for an arbitrary $\boldsymbol{W}$. As such the statement from Wickramasuriya et al. (2019) that "MinT reconciled forecasts are at least as good as the incoherent forecasts" should be qualified this is true only with respect to a loss function that depends on $\boldsymbol{W}$. If Euclidean distance (or mean squared error) is used, there will be realisations where the MinT estimator does 
not improve forecast accuracy relative to base forecasts. This will be demonstrated using a real data set in the empirical study in Section 5.2.

\subsection{MinT}

While the properties discussed so far hold for any projection matrix, the MinT method of Wickramasuriya et al. (2019) has an additional optimality property. Wickramasuriya et al. (2019) show that for unbiased base forecasts, the trace of the forecast error covariance matrix of reconciled forecasts is minimised by an oblique projection with a particular choice of $\boldsymbol{W}$. Their choice is that $\boldsymbol{W}$ should be the forecast error covariance matrix where errors come from using the base forecasts. Although the base forecast error covariance matrix is unknown, it can be estimated using in-sample errors.

Figure 4 provides geometrical intuition into the MinT method. Suppose that the orange points in panel (a) represent in-sample forecast errors. These provide information on the most likely direction of large deviations from the coherent subspace $\mathfrak{s}$. This direction is denoted by $\boldsymbol{R}$. Panel (b) shows a target value of $\boldsymbol{y}$, while the grey points indicate possible values for the base forecasts (the base forecasts are of course stochastic). One possible value of the forecast is depicted in blue as $\hat{\boldsymbol{y}}$. An oblique projection of the blue point back along

the direction of $\boldsymbol{R}$, yields a reconciled forecast closer to the target, especially compared to an orthogonal projection. Panel (c) shows the orthogonal projection of every potential base forecast onto the coherent subspace. Panel (d) depicts an oblique projection along $\boldsymbol{R}$ for all the gray points. The oblique projection yields reconciled forecasts tightly packed near the target $\boldsymbol{y}$. In this sense, the oblique MinT projection minimises the forecast error variance of the reconciled forecasts. In contrast to the result in Theorem 3.2, this property is a statistical property in the sense that MinT is optimal in expectation. 


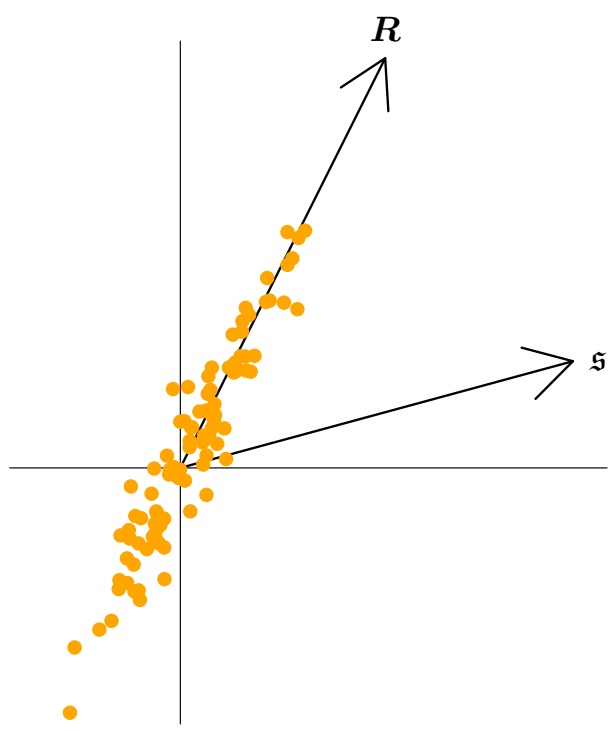

(a)

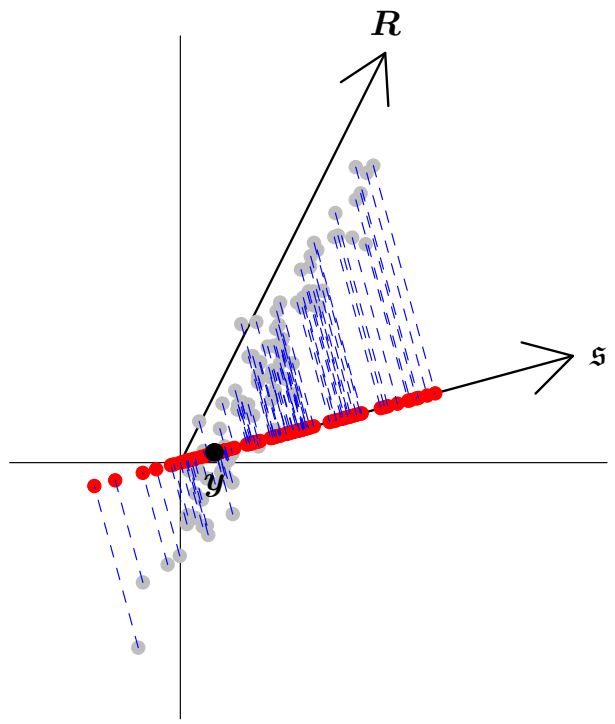

(c)

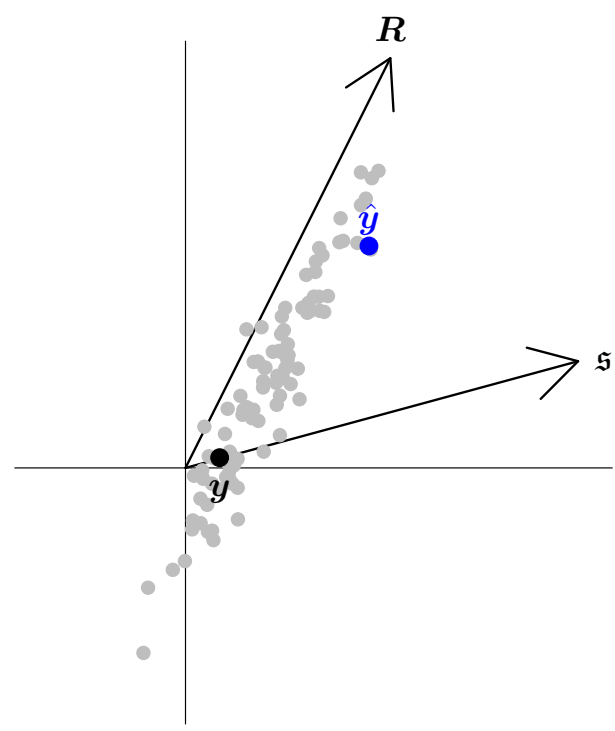

(b)

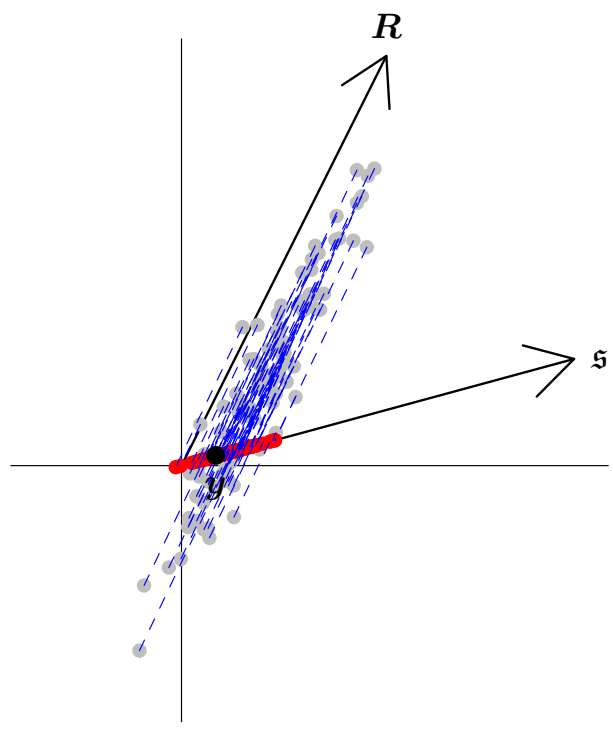

(d)

Figure 4: A schematic represention of orthogonal and oblique reconciliations. The orange points in (a) represent in-sample errors and $\boldsymbol{R}$ shows the most likely direction of deviations from the coherent subspace $\mathfrak{s}$. Grey points in (b) indicate potential base forecasts while the blue dot $\hat{\boldsymbol{y}}$ represents one such realisation. The black dot $\boldsymbol{y}$ denotes the (unknown) target of the forecast. (c) shows the orthogonal projection of all potential base forecasts onto the coherent subspace while (d) shows an oblique projection. 


\section{Bias in forecast reconciliation}

Before turning our attention to the issue of bias itself it is important to state a desirable property that any reconciliation method should have. That is if base forecasts are already coherent then reconciliation should not change the forecasts. As stated in Section 3, this property holds only when $\boldsymbol{S G}$ is a projection matrix. As a corollary, reconciling using an arbitrary $\boldsymbol{G}$, may in fact change an already coherent forecast.

The property that projections map all vectors in the coherent subspace onto themselves is also useful in proving the unbiasedness preserving property of reconciliation of Wickramasuriya et al. (2019). Before restating this proof using a clear geometric interpretation, we discuss in a precise fashion what is meant by unbiasedness.

Suppose that the target of a point forecast is $\boldsymbol{\mu}_{t+h \mid t}:=\mathrm{E}\left(\boldsymbol{y}_{t+h} \mid \boldsymbol{y}_{1}, \ldots, \boldsymbol{y}_{t}\right)$ where the expectation is taken over the predictive density. Our point forecast can be thought of as an estimate of this quantity. The forecast is random due to uncertainty in the training sample and it is with respect to this uncertainty that unbiasedness is defined. Specifically, the point forecast will be unbiased if $\mathrm{E}_{1: t}\left(\hat{\boldsymbol{y}}_{t+h \mid t}\right)=\boldsymbol{\mu}_{t+h \mid t}$, where the subscript $1: t$ denotes an expectation taken over the training sample.

Theorem 4.1 (Unbiasedness preserving property). For unbiased $\hat{\boldsymbol{y}}_{t+h \mid t}$, the reconciled point forecast is also an unbiased prediction as long as $\boldsymbol{S G}$ is a projection onto $\mathfrak{s}$.

Proof. The expected value of the reconciled forecast is given by

$$
\mathrm{E}_{1: t}\left(\tilde{\boldsymbol{y}}_{t+h \mid t}\right)=\mathrm{E}_{1: t}\left(\boldsymbol{S} \boldsymbol{G} \hat{\boldsymbol{y}}_{t+h \mid t}\right)=\boldsymbol{S} \boldsymbol{G} \mathrm{E}_{1: t}\left(\hat{\boldsymbol{y}}_{t+h \mid t}\right)=\boldsymbol{S} \boldsymbol{G} \boldsymbol{\mu}_{t+h \mid t} .
$$

Since $\boldsymbol{\mu}_{t+h \mid t}$ is an expectation taken with respect to the degenerate predictive density it must lie in $\mathfrak{s}$. We have already established that when $\boldsymbol{S G}$ is a projection onto $\mathfrak{s}$ then it maps all vectors in $\mathfrak{s}$ onto themselves. As such $\boldsymbol{S} \boldsymbol{G} \boldsymbol{\mu}_{t+h \mid t}=\boldsymbol{\mu}_{t+h \mid t}$ when $\boldsymbol{S} \boldsymbol{G}$ is a projection matrix.

The above result holds when the projection $\boldsymbol{S G}$ has the coherent subspace $\mathfrak{s}$ as its image and not for all projection matrices in general. To describe this more explicitly suppose $\boldsymbol{S G}$ has as its image $\mathfrak{L}$ which is itself a lower dimensional linear subspace of $\mathfrak{s}$, i.e. $\mathfrak{L} \subset \mathfrak{s}$. Then 
for $\left\{\boldsymbol{\mu}_{t+h \mid t}: \boldsymbol{\mu}_{t+h \mid t} \in \mathfrak{s}, \boldsymbol{\mu}_{t+h \mid t} \notin \mathfrak{L}\right\}, \boldsymbol{S G} \boldsymbol{\mu}_{t+h \mid t} \neq \boldsymbol{\mu}_{t+h \mid t}$. This is depicted in Figure 5 where $\boldsymbol{\mu}$ is projected to a point $\boldsymbol{\mu}^{*}$ in $\mathfrak{L}$. In this case, the expectation of reconciled forecast will be $\boldsymbol{\mu}^{*}$ rather than $\boldsymbol{\mu}$ and hence biased.

This result has implications in practice. The top-down method (Gross \& Sohl 1990) has

$$
\boldsymbol{G}=\left(\begin{array}{ll}
\boldsymbol{p} & \mathbf{0}_{(m \times n-1)}
\end{array}\right),
$$

where $\boldsymbol{p}=\left(p_{1}, \ldots, p_{m}\right)^{\prime}$ is an $m$-dimensional vector consisting a set of proportions used to disaggregate the top-level forecast. In this case it can be verified that $\boldsymbol{S} \boldsymbol{G}$ is idempotent, i,e. $\boldsymbol{S} \boldsymbol{G} \boldsymbol{G}=\boldsymbol{S} \boldsymbol{G}$ and therefore $\boldsymbol{S} \boldsymbol{G}$ is a projection matrix. However the image of this projection is not an $m$-dimensional subspace but a 1-dimensional subspace. As such, topdown reconciliation produces biased forecasts even when the base forecasts are unbiased.

Finally, it is often stated that an assumption required to prove the unbiasedness preserving property is that $\boldsymbol{S G S}=\boldsymbol{S}$ or alternatively that $\boldsymbol{G} \boldsymbol{S}=\boldsymbol{I}$. Both of these conditions are equivalent to assuming that $\boldsymbol{S} \boldsymbol{G}$ is a projection matrix (see Section A.1 in Appendix A for a proof). However, problems arise when viewing the preservation of unbiasedness through the prism of imposing the constraint $\boldsymbol{G S}=\boldsymbol{I}$. This thinking suggests that a way to deal with biased forecasts is to select $\boldsymbol{G}$ in an unconstrained manner. However, equipped with a geometric understanding of the problem, we would advise against this approach. The constraint $\boldsymbol{G} \boldsymbol{S}=\boldsymbol{I}$ is not just about bias. Dropping the constraint compromises all of the attractive properties of projections. It also opens the door to reconciliation methods that change already coherent base forecasts, which suggests an increase in the variability of the forecasts. This seems particularly perverse when the motivation for using a biased method in the first place is to reduce variance.

\subsection{Bias correction}

Our own solution to dealing with biased forecasts is to bias correct before reconciliation. In many cases the method for bias correction will be context specific. For instance, in our empirical study in Section 5 we consider a scenario where bias is induced via taking either a log transformation or a Box-Cox transformation before modelling. In this well-known 


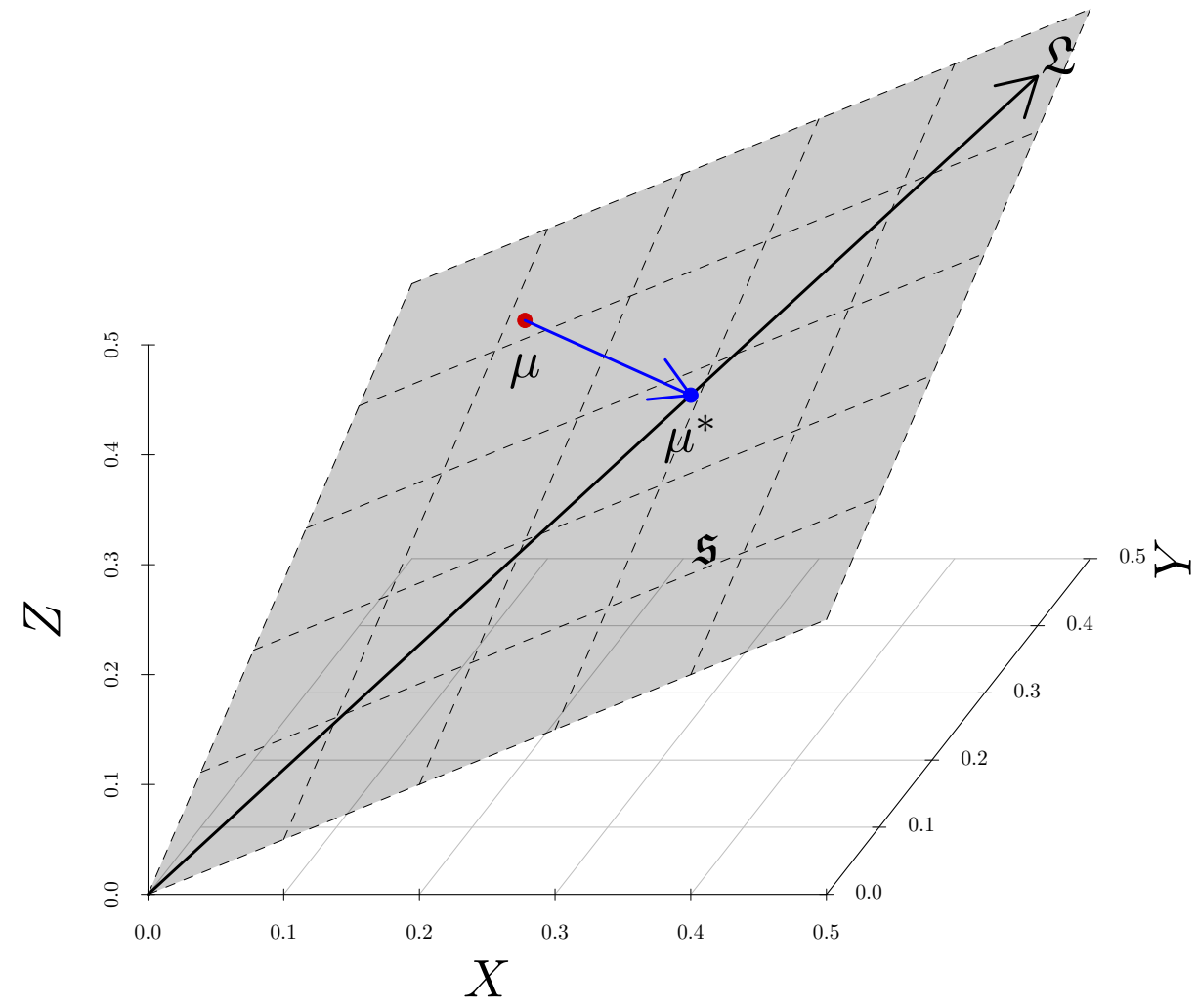

Figure 5: $\mathfrak{L}$ is a linear subspace of the coherent subspace $\mathfrak{s}$. If a projection is onto $\mathfrak{L}$ instead of $\mathfrak{s}$, then $\boldsymbol{\mu} \in \mathfrak{s}$ will be moved to $\boldsymbol{\mu}^{*} \in \mathfrak{L}$.

case a number of bias correction methods exist based on Taylor expansions.

Alternatively, a more general purpose approach to bias correction is to simply estimate the bias by taking the sample mean of $\boldsymbol{y}_{t+h}-\hat{\boldsymbol{y}}_{t+h \mid t}$ for all $t+h$ in the training sample. This can then be subtracted from future forecasts. As stated in the discussion of MinT, in-sample errors are already used to estimate the optimal direction of projection. As such it may be possible to use the same errors to bias correct. Geometrically, the intuition is simple. In panel (a) of Figure 4, the orange points are centered around the origin as would be expected from an unbiased forecast. If forecasts are biased, then errors should simply be translated until they are centered at the origin. Nonetheless there are also a number of pitfalls to such an approach. First, for the very construction we consider, 
where bias is induced by taking a log or Box Cox transformation, bias should be corrected by a multiplicative rather than an additive factor. Second, if in-sample errors are nonstationary due to model misspecification or structural breaks, then the proposed method for bias correction may break down.

\section{$5 \quad$ Empirical study}

Using an empirical application to forecast Australian domestic tourism flows, we illustrate the usefulness of projection-based reconciliation in practice. Previous studies have found that reconciliation improves point forecast accuracy in domestic tourism flows for Australia (see for example Athanasopoulos et al. 2009, Hyndman et al. 2011, Wickramasuriya et al. 2019). However, our motivation in this study is twofold. First, we demonstrate the implications of Theorem 3.1 by comparing reconciled and base forecasts. In contrast to previous studies, we consider individual periods rather than computing averages over a rolling window. Second, we demonstrate how the bias correction methods discussed in the previous section along with the projection-based reconciliation help to improve forecast accuracy.

\subsection{Data}

We consider "overnight trips" across Australia as a measure of domestic tourism flows. The data are provided by the National Visitor Survey and are collected through telephone interviews from an annual sample of 120,000 Australian residents aged 15 years or more. We disaggregate tourism flows into 7 states, 27 zones and 75 regions forming a natural geographical hierarchy that is of interest to tourism operators and policy makers amongst others. Hence, there are 110 series across the hierarchy with 75 bottom-level series. More information about the series and the geographical hierarchy is presented in Table 3 in Appendix B. The data span the period January 1998 to December 2017, which gives a total of 240 observations per series.

Figure 6 shows time, sub-series and seasonal plots of the aggregate overnight trips. As is usual with tourism data, these show a strong seasonal pattern with peaks observed every 
January corresponding to the summer vacation season in Australia. There are also some lower peaks observed in April, July and October corresponding to school term breaks. On the other hand, the month with the least overnight trips is February indicating that people travel least for the month following their summer vacation. The time plot also shows a pronounced upward trend starting from around 2010 to the end of the sample, with flows being fairly flat from the beginning of the sample and a slight downward trend during 2004-2010.

The top panel of Figure 7 shows time plots for the seven states, hence the first level of the geographical hierarchy. The panels below show some selected series from the secondlevel zones and the bottom-level regions. The plots display the diversity of time series features, within but also between levels. For example, noticeable at the first level is the asynchronous seasonal pattern between the Northern Territory and the other states. For

the Northern Territory the high tourist season occurs during June-August with July being the peak, while the low season is during December-February. This reflects the tropical climate of the Northern Territory, with Australians mostly visiting the North during its dry winter-season rather than the wet summer season. Noticeable as we move to the lower levels is the variation in the signal-to-noise ratio, with the regional bottom-level series being much noisier compared to the series from levels above. This of course highlights the importance of modelling series at all levels without any loss of valuable information. We should note here that we observed an anomalous (extremely high) observation for 'Adelaide Hills' for December 2002. We replaced this observation with the average overnight trips on December 2001 and December 2003 for the same destination.

\subsection{Comparison to Base Forecasts}

To demonstrate the implications of Theorem 3.1 we consider the improvement of different reconciliation methods over base forecasts. For each series the ARIMA model minimising AICc is chosen using the auto.arima() function in the forecast package. Using these fitted models, base forecasts are produced for $h=1$ to 12-steps ahead for each series in the hierarchy. This is first carried out with a training sample of 100 observations, i.e., Jan- 

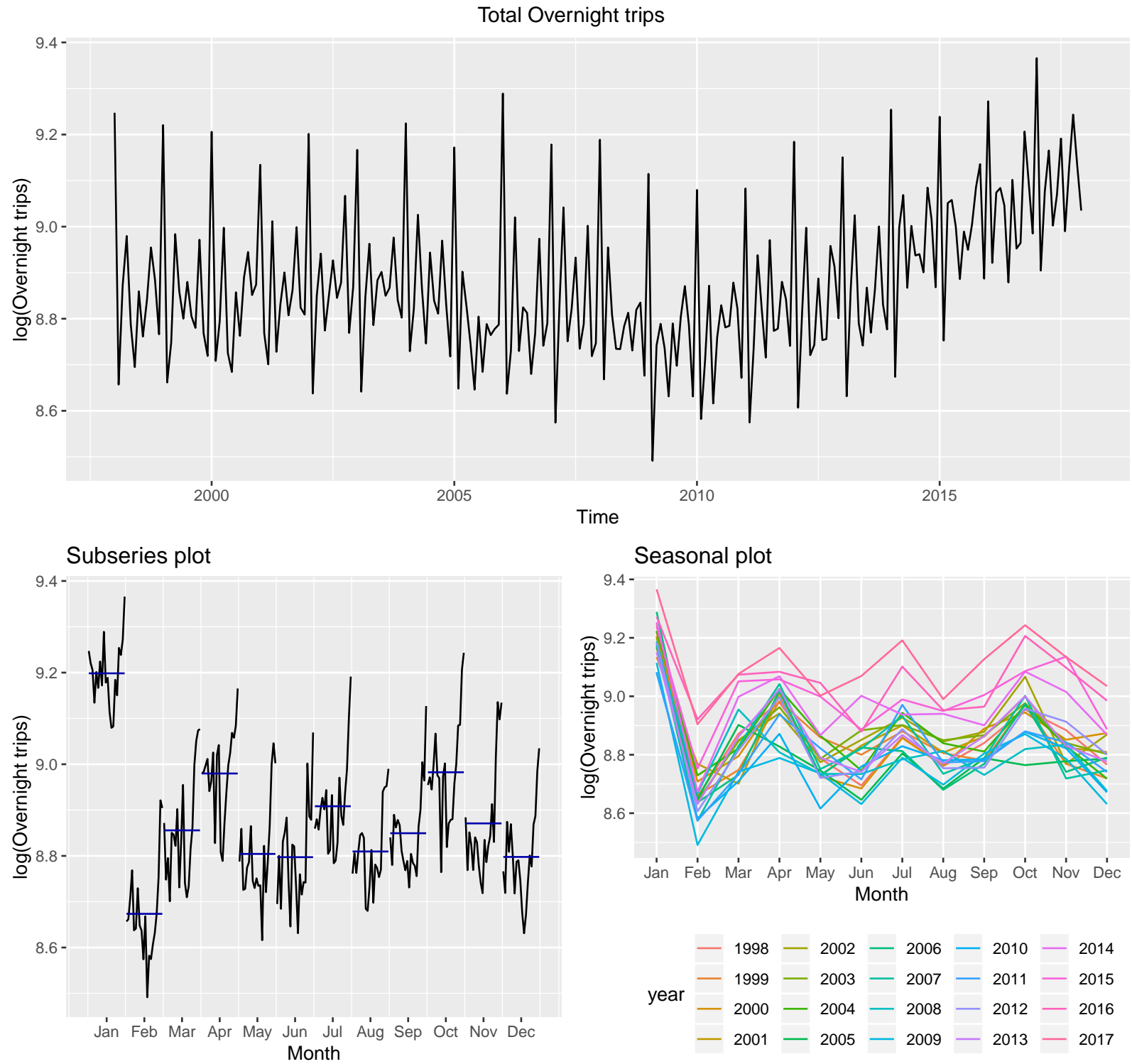

Figure 6: Total domestic overnight trips (in logs) for Australia from January 1998 to December 2017. The top-panel shows a time plot; the bottom-left panel a sub-series plot for each month; the bottom-right panel shows a seasonal plot coloured by year. 
States

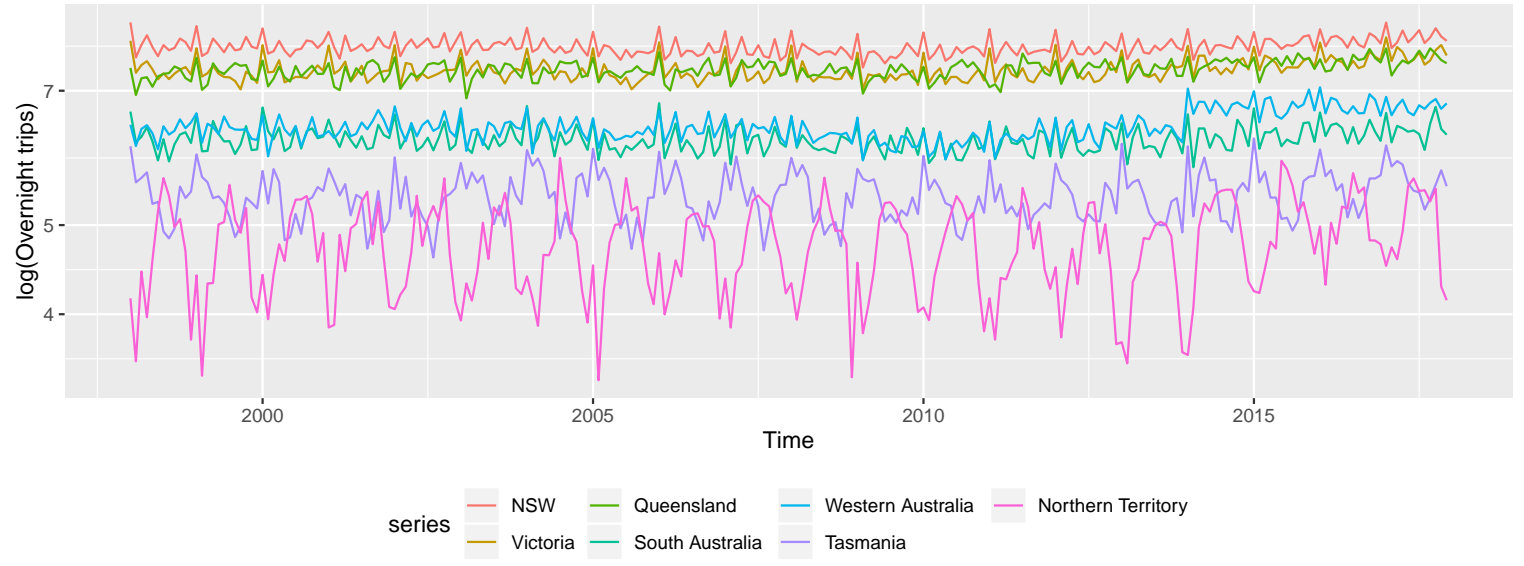

Zones
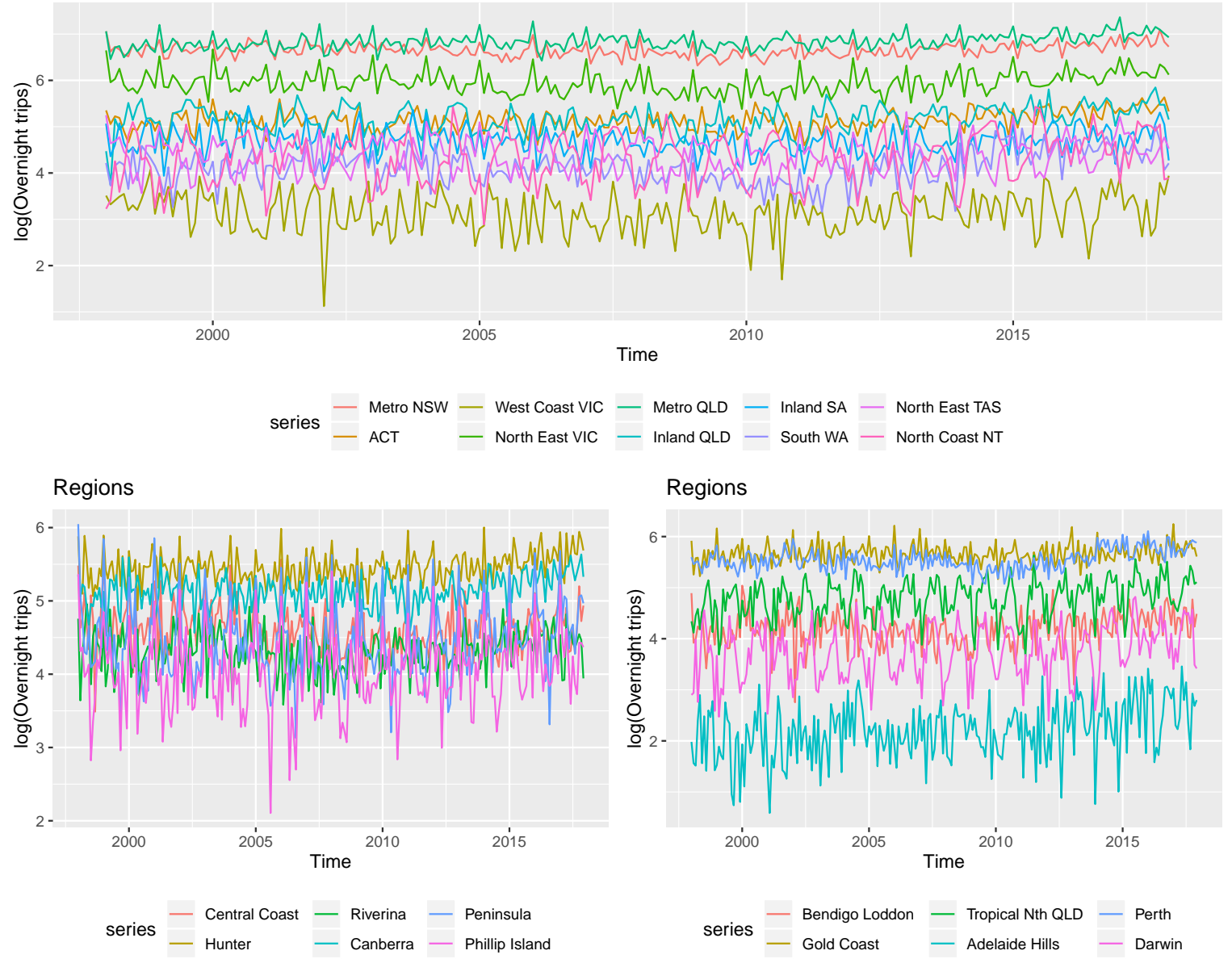

Regions

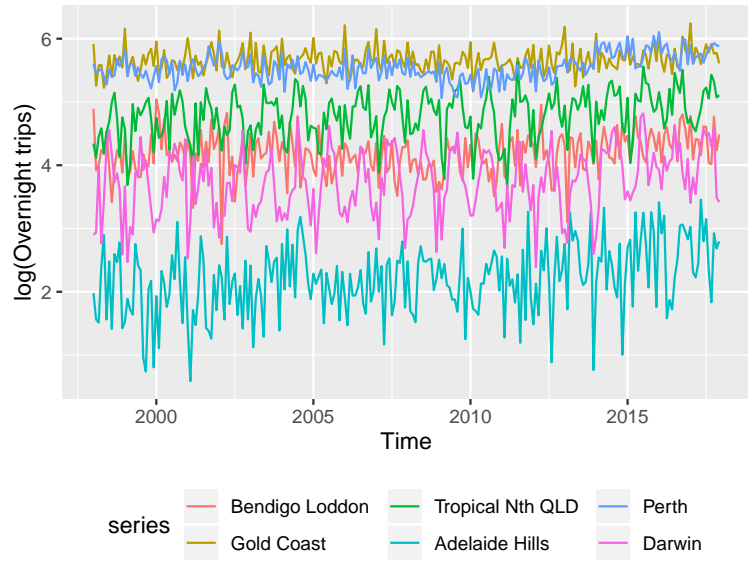

Figure 7: Time plot of overnight trips for some selected series from different disaggregate levels of the hierarchy. All values are presented in log scale. To avoid impact from the zero values we added a constant 1 to all observations 
1998 to Apr-2006. The training window is then rolled forward one observation at a time until the end of the sample. This generates 140 1-step-ahead, 139 2-steps-ahead through to 129 12-steps-ahead forecasts available for forecast evaluation.

After obtaining the base forecasts these are reconciled using three different projection methods: OLS reconciliation, MinT using a shrinkage estimator for $\boldsymbol{W}$ and WLS reconciliation using variance scaling. Squared forecast errors are computed for each series and aggregated across the entire hierarchy; i.e., we compute the loss function described in Section 3.1. We then compute the difference in this loss function between base forecasts and reconciled forecasts, where positive values indicate that reconciled forecasts are more accurate. We also include bottom-up forecasts in this comparison for completeness.

The boxplots in Figure 8 summarise the distribution of this measure over each rolling window. We only present the results for $h=1$, but the results and conclusions that follow are almost identical for the other longer forecast horizons. We do not present these here to save space but they are available upon request.

As the theory predicts, OLS reconciliation always leads to an improvement relative to base forecasts. The entire boxplot for OLS reconciliation is above zero. This is not the case for MinT and WLS which for some windows generate forecasts less accurate than the base forecasts. The boxplots also demonstrate that OLS reconciliation is more stable than MinT and WLS reconciliation, a result not entirely unsurprising since the latter two methods require estimation of a $\boldsymbol{W}$ matrix which in practice is not trivial. Nonetheless when averaging over the entire rolling window the MinT estimator performs best on average, which is again precisely what the theorem proved in Wickramasuriya et al. (2019) would suggest. The average MSE values across the entire rolling window of 140 replications are shown in Table 1.

Table 1: Average $\operatorname{MSE}\left(\times 10^{3}\right)$ over all 140 rolling window replications for $h=1$.

\begin{tabular}{cccccc}
\hline & Base & OLS & MinT & WLS & Bottom-up \\
MSE & 4.31 & 4.20 & $\mathbf{4 . 1 6}$ & 4.48 & 5.28 \\
\hline
\end{tabular}




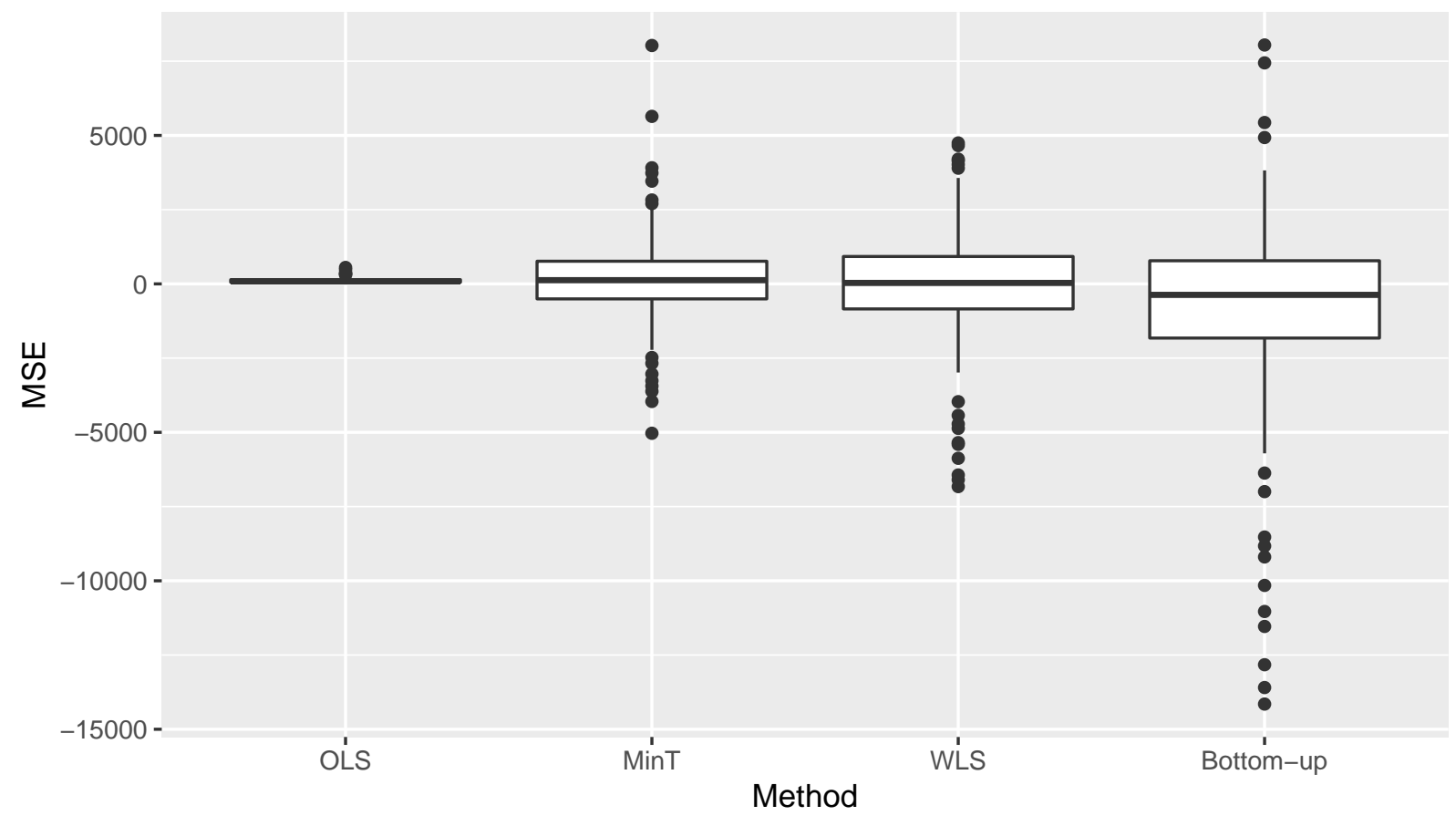

Figure 8: MSE differences between base forecasts and reconciled forecasts using OLS, MinT and WLS, and also the bottom-up approach for $h=1$. A positive value indicates that the coherent forecasts are an improvement over the base forecasts.

\subsection{Transformations and bias adjustment}

We first transform each series in the hierarchy using two types of transformations. Namely, we perform a log-transformation and also the more general Box-Cox transformation. A Box-Cox transformation is defined as,

$$
w_{t}= \begin{cases}\log \left(y_{t}\right) & \text { if } \lambda=0 \\ \frac{y_{t}^{\lambda}-1}{\lambda} & \text { otherwise. }\end{cases}
$$

We first set $\lambda=0$ and hence consider only a log transformation. For the second more general Box-Cox transformation we select $\lambda$ using the "Guerrero" method (Guerrero 1993) implemented in the BoxCox.lambda() function in the forecast package in R (Hyndman et al. 2019). In order to avoid extreme and volatile transformations we restrict $\lambda \in(-0.5,2)$. As zero observations exist in some of the bottom-level series, before transforming we add a constant (more specifically 1) to each series. This overcomes the challenge of undefined 
transformed values for zero observations when we specifically implement the log transformation or when $\lambda$ is selected to be zero by the "Guerrero" method. The constant is subtracted from the final forecasts.

After transformation we fit univariate ARIMA models to each transformed series. The auto.arima() function in the forecast package is used to choose the best model that minimises the AICc. Using the fitted models, forecasts are produced for $h=1$ to 12 -steps ahead for each series in the hierarchy. The same rolling window described in Section 5.2 is used here as well.

The forecasts are then back-transformed by simply reversing the Box-Cox transformation using,

$$
\hat{y}_{t+h \mid t}= \begin{cases}\exp \left(\hat{w}_{t+h \mid t}\right) & \text { if } \lambda=0 \\ \left(\lambda \hat{w}_{t+h \mid t}+1\right)^{1 / \lambda} & \text { otherwise. }\end{cases}
$$

These back-transformed forecasts are potentially biased as they are not the mean of the forecast distribution but the median (assuming that the distribution of the transformed space is symmetric). Hence, the reconciled forecasts that follow from these forecasts will also be biased. We refer to these as "Biased" base forecasts in the results that follow. This is the exact scenario we want to demonstrate in this study and we next move to our proposed solution of bias correcting the base forecasts before reconciling for which we explore two scenarios.

Using a Taylor series expansion (Guerrero 1993), the back-transformed mean of the forecast distribution for a Box-Cox transformation is given by

$$
\hat{y}_{t+h \mid t}= \begin{cases}\exp \left(\hat{w}_{t+h \mid t}\right)\left[1+\frac{\sigma_{h}^{2}}{2}\right] & \text { if } \lambda=0, \\ \left(\lambda \hat{w}_{t+h \mid t}+1\right)^{1 / \lambda}\left[1+\frac{\sigma_{h}^{2}(1-\lambda)}{2\left(\lambda \hat{w}_{t+h \mid t}+1\right)^{2}}\right] & \text { if } \lambda \neq 0,\end{cases}
$$

where $\hat{w}_{t+h \mid t}$ is the $h$-step-ahead forecast from the Box-Cox transformed series and $\sigma_{h}^{2}$ is the variance of $\hat{w}_{t+h \mid t}$. Using the mean of the forecast distribution returns bias-adjusted base forecasts compared to the simple back-transformation of Eq. (1). We refer to this as "Method-1" in the results that follow. The second scenario of bias adjustment we explore is using the in-sample forecast error mean of the biased forecasts to adjust the out-of-sample forecasts. We refer to this as "Method-2" in the results that follow. 
Using the three sets of base forecasts from each of the two transformations, we generate coherent forecasts implementing OLS, MinT and WLS reconciliation projections, and also the bottom-up approach and compare the results for when the base forecasts are biased and bias-adjusted, i.e., unbiased. Table 2 presents the results over the 140 rolling window replications for 1-step-ahead forecasts. As was the case with Section 5.2, the conclusions that follow extend almost identically to longer forecast horizons. We do not present these here to save space but they are available upon request.

Recall that reconciliation approaches via projections preserve unbiasedness in the reconciled forecasts iff the base forecasts are unbiased. Hence, the two columns labelled "Biased" contain results for biased base but also reconciled forecasts. Using Method-1 for first bias adjusting the base forecasts and then reconciling, results in forecast improvements for all methods for both the log and the Box-Cox transformation induced bias. In this case MinT returns the lowest MSE across all forecasts. Notice that the "Box-Cox biased" base forecasts carry a higher bias compared to the "log biased" base forecasts. Even for these MinT

Table 2: Average MSE $\left(\times 10^{3}\right)$ of 1-step-ahead point forecasts from log and Box-Cox transformed series. Biased denotes forecasts from simply reversing the transformation via Eq. (1). Unbiased(Method-1) performs bias adjustment via a Taylor series expansion as shown in Eq. (2) whereas Unbiased(Method-2) bias adjusts by subtracting the in-sample forecast error mean.

\begin{tabular}{lccccccc}
\hline & \multicolumn{3}{c}{ Log Transformation } & & \multicolumn{2}{c}{ Box-Cox Transformation } \\
\cline { 2 - 3 } Method & Biased & $\begin{array}{c}\text { Unbiased } \\
\text { (Method-1) }\end{array}$ & $\begin{array}{c}\text { Unbiased } \\
\text { (Method-2) }\end{array}$ & & Biased & $\begin{array}{c}\text { Unbiased } \\
\text { (Method-1) }\end{array}$ & $\begin{array}{c}\text { Unbiased } \\
\text { (Method-2) }\end{array}$ \\
\hline Base & 4.47 & 4.43 & 4.51 & & 4.59 & 4.52 & 4.78 \\
OLS & 4.34 & 4.31 & 4.36 & & 4.46 & 4.40 & 4.63 \\
MinT & 4.32 & $\mathbf{4 . 1 6}$ & 4.61 & & 4.29 & $\mathbf{4 . 1 7}$ & 4.54 \\
WLS & 4.82 & 4.38 & 5.43 & & 4.77 & 4.42 & 5.23 \\
Bottom-up & 6.36 & 5.26 & 8.06 & & 6.21 & 5.31 & 7.46 \\
\hline
\end{tabular}


reconciliation of bias adjusted base forecasts generates very accurate forecasts, almost as accurate as the forecasts that come from the less biased log transformation.

In contrast to the results from using Method-1 for bias adjusting before reconciliation,

using Method-2 has an adverse effect on the forecast accuracy of the reconciled forecasts. In this case the reconciled unbiased forecasts are less accurate than the biased reconciled forecasts. This reflects the fact and sends the warning that implementing inappropriate bias adjustment, in this case using an additive rather than a multiplicative factor, will hinder forecast accuracy and extra care must be taken in this bias adjustment procedure.

\section{Conclusions}

Defining concepts such as coherence and reconciliation in geometric terms provides new insights into hierarchical forecasting methods. We have also provided evidence that bias correction before reconciliation improves forecast accuracy compared to approaches that do not bias correct and/or do not use reconciliation. Our intention in proposing a geometric interpretation is also to provoke research into new areas. We now discuss three such possibilities.

First, it should be possible to extend the concept of coherence to non-linear constraints. In these cases the coherent space may need to be defined by a manifold. Although much more challenging, it is still possible to define reconciled forecasts in terms of projections onto a manifold. Second, since we have established that the concept of bottom-level series is not crucial in forecast reconciliation, an open question is whether it may be better to construct base forecasts of linear combinations of the time series rather than the time series themselves. Finally, the geometric interpretations of hierarchical forecast reconciliation facilitates an extension into a probabilistic framework. The latter two are issues we investigate in separate papers. 


\section{A Appendix}

\section{A.1 Proof $S G S=S$ implies $S G$ is a projection}

Here we establish that if $\boldsymbol{S} \boldsymbol{G}$ is a projection onto the linear subspace spanned by $\boldsymbol{S}$ then $\boldsymbol{S G S}=\boldsymbol{S}$. We also prove that the converse holds, namely that if the condition $\boldsymbol{S} \boldsymbol{G} \boldsymbol{S}=\boldsymbol{S}$ holds then $\boldsymbol{S} \boldsymbol{G}$ must be a projection onto the linear subspace spanned by $\boldsymbol{S}$.

To establish the first statement, let $\boldsymbol{s}_{j}$ be the $j$ th column of $\boldsymbol{S}$. Since by definition, $\boldsymbol{s}_{j}$ lies in $\mathfrak{s}$, it must hold that $\boldsymbol{S G} \boldsymbol{s}_{j}=\boldsymbol{s}_{j}$. Stacking these vectors horizontally

$$
\begin{aligned}
\boldsymbol{S G S} & =\left(\begin{array}{llll}
\boldsymbol{S G} \boldsymbol{s}_{1}, & \boldsymbol{S G} \boldsymbol{s}_{2}, & \cdots & \boldsymbol{S G} \boldsymbol{s}_{m}
\end{array}\right) \\
& =\left(\begin{array}{llll}
\boldsymbol{s}_{1}, & \boldsymbol{s}_{2}, & \cdots & \boldsymbol{s}_{m}
\end{array}\right) \\
& =\boldsymbol{S}
\end{aligned}
$$

To establish the converse it suffices to postmultiply the condition $\boldsymbol{S} \boldsymbol{G}=\boldsymbol{S}$ by $\boldsymbol{G}$. This yields $\boldsymbol{S} \boldsymbol{G} \boldsymbol{G}=\boldsymbol{S} \boldsymbol{G}$, which in turn implies idempotence since $(\boldsymbol{S} \boldsymbol{G})^{2}=\boldsymbol{S} \boldsymbol{G}$. 


\section{B Australian Tourism Data}

Table 3: Geographical hierarchy of Australian tourism flow

\begin{tabular}{|c|c|c|c|c|c|c|c|c|}
\hline \multicolumn{3}{|c|}{ Level 0 - Total } & \multicolumn{3}{|c|}{ Regions cont. } & \multicolumn{3}{|c|}{ Regions cont. } \\
\hline 1 & Tot & Australia & 37 & $\mathrm{AAB}$ & Central Coast & 76 & $\mathrm{CBD}$ & Mackay \\
\hline \multicolumn{3}{|c|}{ Level 1 - States } & 38 & $\mathrm{ABA}$ & Hunter & 77 & $\mathrm{CCA}$ & Whitsundays \\
\hline 2 & A & NSW & 39 & $\mathrm{ABB}$ & North Coast NSW & 78 & $\mathrm{CCB}$ & Northern \\
\hline 3 & $\mathrm{~B}$ & Victoria & 40 & $\mathrm{ACA}$ & South Coast & 79 & $\mathrm{CCC}$ & Tropical North Queensland \\
\hline 4 & $\mathrm{C}$ & Queensland & 41 & $\mathrm{ADA}$ & Snowy Mountains & 80 & CDA & Darling Downs \\
\hline 5 & $\mathrm{D}$ & South Australia & 42 & $\mathrm{ADB}$ & Capital Country & 81 & $\mathrm{CDB}$ & Outback \\
\hline 6 & $\mathrm{E}$ & Western Australia & 43 & $\mathrm{ADC}$ & The Murray & 82 & DAA & Adelaide \\
\hline 7 & $\mathrm{~F}$ & Tasmania & 44 & $\mathrm{ADD}$ & Riverina & 83 & $\mathrm{DAB}$ & Barossa \\
\hline 8 & G & Northern Territory & 45 & AEA & Central NSW & 84 & DAC & Adelaide Hills \\
\hline \multicolumn{3}{|c|}{ Level 2 - Zones } & 46 & AEB & New England North West & 85 & DBA & Limestone Coast \\
\hline 9 & AA & Metro NSW & 47 & $\mathrm{AEC}$ & Outback NSW & 86 & DBB & Fleurieu Peninsula \\
\hline 10 & $\mathrm{AB}$ & North Coast NSW & 48 & AED & Blue Mountains & 87 & DBC & Kangaroo Island \\
\hline 11 & $\mathrm{AC}$ & South Coast NSW & 49 & AFA & Canberra & 88 & DCA & Murraylands \\
\hline 12 & $\mathrm{AD}$ & South NSW & 50 & BAA & Melbourne & 89 & DCB & Riverland \\
\hline 13 & $\mathrm{AE}$ & North NSW & 51 & $\mathrm{BAB}$ & Peninsula & 90 & $\mathrm{DCC}$ & Clare Valley \\
\hline 14 & $\mathrm{AC}$ & $\mathrm{ACT}$ & 52 & $\mathrm{BAC}$ & Geelong & 91 & DCD & Flinders Range and Outback \\
\hline 15 & $\mathrm{BA}$ & Metro VIC & 53 & $\mathrm{BBA}$ & Western & 92 & DDA & Eyre Peninsula \\
\hline 16 & $\mathrm{BB}$ & West Coast VIC & 54 & $\mathrm{BCA}$ & Lakes & 93 & DDB & Yorke Peninsula \\
\hline 17 & $\mathrm{BC}$ & East Coast VIC & 55 & $\mathrm{BCB}$ & Grippsland & 94 & EAA & Australia's Coral Coast \\
\hline 18 & $\mathrm{BC}$ & North East VIC & 56 & $\mathrm{BCD}$ & Phillip Island & 95 & $\mathrm{EAB}$ & Experience Perth \\
\hline 19 & $\mathrm{BD}$ & North West VIC & 57 & $\mathrm{BDA}$ & Central Murray & 96 & $\mathrm{EAC}$ & Australia's South West \\
\hline 20 & $\mathrm{CA}$ & Metro QLD & 58 & BDB & Goulburn & 97 & EBA & Australia's North West \\
\hline 21 & $\mathrm{CB}$ & Central Coast QLD & 59 & $\mathrm{BDC}$ & High Country & 98 & $\mathrm{ECA}$ & Australia's Golden Outback \\
\hline 22 & $\mathrm{CC}$ & North Coast QLD & 60 & $\mathrm{BDD}$ & Melbourne East & 99 & FAA & Hobert and South \\
\hline 23 & $\mathrm{CD}$ & Inland QLD & 61 & $\mathrm{BDE}$ & Upper Yarra & 100 & FBA & East Coast \\
\hline 24 & DA & Metro SA & 62 & $\mathrm{BDF}$ & Murray East & 101 & FBB & Launceston, Tamar \& North \\
\hline 25 & DB & South Coast SA & 63 & BEA & Wimmera+Mallee & 102 & $\mathrm{FCA}$ & North West \\
\hline 26 & $\mathrm{DC}$ & Inland SA & 64 & $\mathrm{BEB}$ & Western Grampians & 103 & FCB & Wilderness West \\
\hline 27 & DD & West Coast SA & 65 & $\mathrm{BEC}$ & Bendigo Loddon & 104 & GAA & Darwin \\
\hline 28 & $\mathrm{EA}$ & West Coast WA & 66 & BED & Macedon & 105 & GAB & Kakadu Arnhem \\
\hline 29 & EB & North WA & 67 & $\mathrm{BEE}$ & Spa Country & 106 & GAC & Katherine Daly \\
\hline 30 & $\mathrm{EC}$ & South WA & 68 & $\mathrm{BEF}$ & Ballarat & 107 & GBA & Barkly \\
\hline 31 & FA & South TAS & 69 & $\mathrm{BEG}$ & Central Highlands & 108 & GBB & Lasseter \\
\hline 32 & $\mathrm{FB}$ & North East TAS & 70 & CAA & Gold Coast & 109 & GBC & Alice Springs \\
\hline 33 & $\mathrm{FC}$ & North West TAS & 71 & $\mathrm{CAB}$ & Brisbane & 110 & GBD & MacDonnell \\
\hline 34 & GA & North Coast NT & 72 & $\mathrm{CAC}$ & Sunshine Coast & & & \\
\hline 35 & GB & Central NT & 73 & CBA & Central Queensland & & & \\
\hline \multicolumn{3}{|c|}{ Level 2 - Regions } & 74 & $\mathrm{CBB}$ & Bundaberg & & & \\
\hline 36 & AAA & Sydney & 75 & $\mathrm{CBC}$ & Fraser Coast & & & \\
\hline
\end{tabular}




\section{References}

Almeida, V., Ribeiro, R. \& Gama, J. (2016), Hierarchical time series forecast in electrical grids, in K. J. Kim \& N. Joukov, eds, 'Information Science and Applications', Springer, Singapore, pp. 995-1005.

Athanasopoulos, G., Ahmed, R. A. \& Hyndman, R. J. (2009), 'Hierarchical forecasts for Australian domestic tourism', International Journal of Forecasting 25(1), 146-166.

Athanasopoulos, G., Gamakumara, P., Panagiotelis, A., Hyndman, R. J. \& Affan, M. (2019), Hierarchical forecasting, in P. Fuleky, ed., 'Macroeconomic Forecasting in the Era of Big Data', Springer, Honolulu, chapter 21, pp. 703-733.

Athanasopoulos, G., Hyndman, R. J., Kourentzes, N. \& Petropoulos, F. (2017), 'Forecasting with temporal hierarchies', European Journal of Operational Research 262, 60-74.

Ben Taieb, S., Taylor, J. W. \& Hyndman, R. J. (2017), Coherent probabilistic forecasts for hierarchical time series, in 'Proceedings of the 34th International Conference on Machine Learning', Vol. 70, PMLR, pp. 3348-3357.

Chase, C. W. (2013), 'Using big data to enhance demand-driven forecasting and planning', Journal of Business Forecasting 32(2), 27-32.

Dunn, D. M., Williams, W. H. \& Dechaine, T. L. (1976), 'Aggregate versus subaggregate models in local area forecasting', Journal of American Statistical Association 71(353), 68-71.

Fliedner, G. (2001), 'Hierarchical forecasting: issues and use guidelines', Industrial Management \& Data Systems 101(1), 5-12.

Gross, C. W. \& Sohl, J. E. (1990), 'Disaggregation methods to expedite product line forecasting', Journal of Forecasting 9(3), 233-254.

Guerrero, V. M. (1993), 'Time-series analysis supported by power transformations', Journal of Forecasting 12(1), 37-48. 
Hyndman, R. J., Ahmed, R. A., Athanasopoulos, G. \& Shang, H. L. (2011), 'Optimal combination forecasts for hierarchical time series', Computational Statistics and Data Analysis 55(9), 2579-2589.

Hyndman, R. J. \& Athanasopoulos, G. (2018), Forecasting: principles and practice, 2nd edn, OTexts, Melbourne, Australia.

Hyndman, R. J., Athanasopoulos, G., Bergmeir, C., Caceres, G., Chhay, L., O'Hara-Wild, M., Petropoulos, F., Razbash, S., Wang, E., Yasmeen, F., R Core Team, Ihaka, R., Reid, D., Shaub, D., Tang, Y. \& Zhou, Z. (2019), forecast: Forecasting Functions for Time Series and Linear Models. Version 8.9.

Jeon, J., Panagiotelis, A. \& Petropoulos, F. (2019), 'Probabilistic forecast reconciliation with applications to wind power and electric load', European Journal of Operational Research 279(2), 364-379.

Kahn, K. B. (1998), 'Revisiting top-down versus bottom-up forecasting', The Journal of Business Forecasting 17(2), 14.

Karmy, J. P. \& Maldonado, S. (2019), 'Hierarchical time series forecasting via support vector regression in the European travel retail industry', Expert Systems with Applications 137, 59-73.

Kourentzes, N. \& Athanasopoulos, G. (2019), 'Cross-temporal coherent forecasts for Australian tourism', Annals of Tourism Research 75, 393-409.

Lapide, L. (1998), 'A simple view of top-down vs bottom-up forecasting', Journal of Business Forecasting Methods \& Systems 17, 28-31.

Li, H. \& Tang, Q. (2019), 'Analyzing mortality bond indexes via hierarchical forecast reconciliation', ASTIN Bulletin 24(3), 823-846.

Mahkya, D., Ulama, B. \& Suhartono (2017), 'Hierarchical time series bottom-up approach for forecast the export value in Central Java', Journal of Physics: Conference Series 893(012033). 
Nystrup, P., Lindström, E., Pinson, P. \& Madsen, H. (2019), 'Temporal hierarchies with autocorrelation for load forecasting', European Journal of Operational Research (forthcoming).

Rao, C. R. (1974), 'Projectors, generalized inverses and the BLUE's', Journal of the Royal Statistical Society: Series B (Methodological) 36(3), 442-448.

Schwarzkopf, A. B., Tersine, R. J. \& Morris, J. S. (1988), 'Top-down versus bottom-up forecasting strategies', International Journal of Production Research 26(11), 1833-1843.

Shang, H. L. \& Hyndman, R. J. (2017), 'Grouped functional time series forecasting: An application to age-specific mortality rates', Journal of Computational and Graphical Statistics 26(2), 330-343.

Van Erven, T. \& Cugliari, J. (2015), Game-theoretically optimal reconciliation of contemporaneous hierarchical time series forecasts, in 'Modeling and Stochastic Learning for Forecasting in High Dimensions', Springer, pp. 297-317.

Wickramasuriya, S. L., Athanasopoulos, G. \& Hyndman, R. J. (2019), 'Optimal forecast reconciliation for hierarchical and grouped time series through trace minimization', Journal of the American Statistical Association 114(526), 804-819. 